\title{
INDICADORES DE QUALIDADE DO SOLO SOB DIFERENTES SISTEMAS DE USO NA MESORREGIÃO DO AGRESTE PARAIBANO ${ }^{1}$
}

\author{
GERÔNIMO FERREIRA DA SILVA ${ }^{2 *}$, DJAIL SANTOS ${ }^{3}$, ALEXANDRE PAIVA DA SILVA ${ }^{3}$, JEORGE MEDEIROS \\ DE SOUZA ${ }^{4}$
}

\begin{abstract}
RESUMO - Os sistemas de cultivo modificam os atributos do solo, podendo alterar sua qualidade. O trabalho teve como objetivo avaliar as alterações nos atributos físicos, químicos e biológicos de solos sob sistemas de cultivo orgânico (solos cultivados organicamente há mais de sete anos), em conversão para orgânico e convencional, tendo como referência o solo sob mata, assim como identificar os atributos a serem utilizados como indicadores de qualidade do solo. Foram selecionadas três áreas de propriedades agrícolas, localizadas nos municípios de Areia, Remígio e Lagoa Seca, Paraíba, para a coleta de amostras de solo deformadas, nas camadas de $0-10$ e 10-20 cm. Os dados foram submetidos à ANOVA, Teste de Tukey e Análise de Componentes Principais. Houve redução da densidade do solo (Ds) e aumento da porosidade total (PT) no sistema de cultivo orgânico comparado aos sistemas de cultivo em conversão para orgânico e convencional. Os teores de fósforo e de carbono orgânico (CO), a soma de bases (SB), a capacidade de troca catiônica (CTC), a matéria orgânica particulada leve (MOPL), o carbono da matéria orgânica particulada leve (CMOPL), a respiração edáfica (RE) e o $\mathrm{pH}$ dos solos sob cultivo orgânico foram superiores aos obtidos nos solos sob cultivo convencional. O cultivo em bases orgânicas proporcionou a manutenção da qualidade do solo em condições semelhantes e/ou melhores que a condição de mata e para as condições deste trabalho os indicadores mais adequados para analisar o cultivo em bases orgânicas foram Ds, PT, CO, MOPL, CMOPL, pH, SB, CTC e RE.
\end{abstract}

Palavras-chaves: Solos. Atributos. Utilização e manejo. Avaliação da qualidade.

\section{SOIL QUALITY INDICATORS UNDER DIFFERENT LAND USE SYSTEMS IN THE AGRESTE REGION OF PARAIBA, BRAZIL}

SUMMARY - Cropping systems modify soil attributes and may change its quality.The work had the objective to evaluate the changes in physical, chemical and biological soil under organic farming systems (soils cultivated organically for more than seven years), in conversion to organic and conventional, with reference to the soil under native forest, and identify the attributes used as indicators of soil quality. Three areas of farm crops, located in the municipalities of Areia, Remígio, and Lagoa Seca, the state of Paraíba, Brazil were selected, and soil samples deformed collected in the layers $0-10$ and $10-20 \mathrm{~cm}$. The data were analyzed using ANOVA, the Tukey Test, and the Principal Component Analysis. There was a reduction of soil density (Ds) and increased porosity (PT) in the system of organic farming compared to cropping systems in conversion organic and conventional. The contents of phosphorus and organic carbon (CO), the base sum (SB), cation exchange capacity (CTC), light particulate organic matter (MOPL), carbon particulate organic matter light (CMOPL), edaphic respiration (RE) and $\mathrm{pH}$ of soils under organic cultivation are higher than soils under conventional tillage.The growing organic basis contributes to the maintenance of soil quality under similar conditions and/or even better than the condition forest and, under the conditions of this study, the most appropriate indicators to analyze the cultivation organic basesare Ds, PT, CO, MOPL, CMOPL, pH, SB, CTC e RE.

Keywords: Soils. Attributes. Use and management soil. Quality soil assessment.

\footnotetext{
*Autor para correspondência

${ }^{1}$ Recebido para publicação em 08/03/2014; aceito em 24/04/2015.

Parte da Dissertação de Mestrado em Manejo de Solo e Água do primeiro autor.

${ }^{2}$ Departamento de Engenharia Agrícola/UFRPE, Rua Dom Manoel de Medeiros, s/n, Dois Irmãos, 52.171-900, Recife (PE), Brasil; agrogefe@yahoo.com.br.

${ }^{3}$ Departamento de Solos e Engenharia Rural, UFPB, Campus II, 58.397-000, Areia (PB), Brasil; santosdj@cca.ufpb.br, paivadasilva@gmail.com.br.

${ }^{4}$ Mestre em Ciência do Solo, Universidade Federal da Paraíba, Areia (PB), Brasil; jeorgecn@yahoo.com.br.
} 


\section{INTRODUÇÃO}

As práticas de manejo do solo provocam alterações nos seus atributos físicos, químicos e biológicos, significando perda de qualidade e afetando a sustentabilidade ambiental e econômica da atividade agrícola (NIERO et al., 2010). Por outro lado, a boa qualidade desses atributos propicia condições adequadas para o crescimento e o desenvolvimento das plantas e para a manutenção da diversidade de organismos que habitam o solo (DORAN; PARKIN, 1994).

Qualquer alteração no solo pode alterar diretamente sua estrutura e atividade biológica e, consequentemente, sua fertilidade, com reflexos nos agroecossistemas, podendo promover prejuízos à sua qualidade e à produtividade das culturas (CARNEIRO et al., 2009). Sendo assim, a compreensão e a quantificação do impacto do uso e manejo do solo na sua qualidade são fundamentais no desenvolvimento de sistemas agrícolas sustentáveis (BAVOSO et al., 2010).

Nesse sentido, o nível de alteração na qualidade do solo pode ser avaliado pela mensuração do estado atual de determinados atributos em comparação com o estado natural do solo, sem interferência antrópica, ou com valores considerados ideais. Para isso, faz-se necessário o uso de um conjunto mínimo de indicadores que apresentem características como facilidade de avaliação, aplicabilidade em diferentes escalas, utilização abrangente e sensibilidade a variações de manejo (DORAN; PARKIN, 1994; NIERO et al., 2010; CHAVES et al., 2012).

$\mathrm{O}$ interesse por pesquisas que visam avaliar as alterações das propriedades físicas, químicas e biológicas do solo durante a fase de transição do cultivo convencional para orgânico, tendo como referência o solo sem interferência antrópica ou sob condições naturais, aumentou consideravelmente nos últimos anos (MARINARI et al., 2006; LIMA et al., 2007). Em todas essas pesquisas é comum a sugestão de avaliação após certo período de tempo de cultivo, pois a percepção de mudanças significativas no solo está na dependência das condições de clima, tipo de manejo e solo.

Na Mesorregião do Agreste da Paraíba, até então, não se tem informação sobre estudos de identificação de indicadores de alterações químicas, físicas e biológicas em áreas sob cultivo orgânico, comparativamente àquelas sob cultivo convencional e em conversão para orgânico, tendo como referência o solo sob condições naturais.

Estudos dessa natureza são importantes, pois podem fornecer um conjunto de informações preciosas, representando um inexplorado potencial para o monitoramento e avaliação da qualidade do solo em diferentes sistemas locais de cultivo e manejo do solo. Estes estudos são, também, de suma importância para uma melhor compreensão da sustentabilidade dos sistemas de cultivo orgânico, a partir da defi- nição de um conjunto de dados mínimos que possam servir como referência para avaliação e seleção de indicadores de qualidade do solo.

Diante do exposto, objetivou-se com este trabalho avaliar as alterações nos atributos físicos, químicos e biológicos de solos sob sistemas de cultivo orgânico (solos cultivados organicamente há mais de sete anos), em conversão para orgânico e convencional, tendo como referência o solo sob condições naturais (mata nativa), assim como identificar os atributos a serem utilizados como indicadores de qualidade do solo.

\section{MATERIAL E MÉTODOS}

O trabalho foi desenvolvido nos municípios de Areia, Remígio e Lagoa Seca, localizados na Mesorregião do Agreste da Paraíba, porção oriental do Estado. Pela classificação de Köppen, o clima das áreas estudadas é do tipo As' (clima quente e úmido, com ocorrência das maiores precipitações nos meses de abril a junho). As temperaturas médias variam entre mínimas de 17 a $20^{\circ} \mathrm{C}$ e máximas de 22 a $26^{\circ}$ C. A umidade relativa do ar situa-se em torno de $79 \%$. As precipitações pluviométricas médias anuais das áreas variam entre 700 e $1600 \mathrm{~mm}$. E as três propriedades localizadas nos municípios de Areia, Remígio e Lagoa Seca foram objetos de estudo, sendo os solos dessas três áreas classificado como Argissolo Vermelho-Amarelo (PVAe) para o município de Areia e Neossolo Regolítico (RRe) para os municípios de Remígio e Lagoa Seca (Embrapa, 2006).

Foram selecionadas três áreas de propriedades agrícolas, caracterizadas pela exploração de sistemas de cultivo orgânico (solos cultivados organicamente há mais de sete anos), em conversão para orgânico (solos com até sete anos de cultivo orgânico) e convencional, além de área com mata nativa, localizadas, respectivamente, nos três municípios acima citados, levando-se em consideração a proximidade das áreas experimentais (para evitar a variabilidade do solo), o tempo de cultivo (o maior tempo de cultivo permite a maior diferenciação entre tratamentos) e uso do solo (cultivos orgânico, em conversão para orgânico, convencional e mata nativa).

A área I (Areia-PB) foi formada por uma propriedade cultivada de forma orgânica há oito anos. Para o cultivo em conversão levou-se em consideração a área utilizada há apenas dois anos de cultivo orgânico. Em área contígua a essa propriedade encontra-se uma outra cultivada de forma convencional com culturas anuais e hortaliças folhosas há mais de oito anos. A mata nativa está situada nos arredores da área com cultivo orgânico. A área II (RemígioPB) foi constituída por uma propriedade cultivada organicamente há quarenta anos com agricultura de sequeiro. A área em conversão, situada a $100 \mathrm{~m}$ da área orgânica, vem sendo cultivada há mais de cinco anos e o cultivo convencional, situado a $500 \mathrm{~m}$ do 
orgânico, vem sendo cultivado há mais de doze anos. $\mathrm{A}$ área de mata nativa está situada a $400 \mathrm{~m}$ do cultivo orgânico. E a área III (Lagoa Seca-PB) foi formada por uma propriedade que cultiva organicamente há nove anos com agricultura de sequeiro. $\mathrm{O}$ cultivo em conversão, situado em área vizinha ao cultivo orgânico, ocorre há seis anos. O cultivo convencional está situado a $800 \mathrm{~m}$ do cultivo orgânico. E a área de mata nativa situa-se a $350 \mathrm{~m}$ do cultivo convencional.

Nas áreas selecionadas das propriedades e nas áreas de mata foram coletadas amostras deformadas de solo para as análises físicas, químicas e biológicas nas camadas de 0-10 e 10-20 cm. A escolha dessas camadas fundamentou-se no fato de que o maior volume do sistema radicular das culturas e as maiores alterações químicas, físicas e biológicas em solos cultivados em plantio convencional ocorrem na camada de $0-20 \mathrm{~cm}$ de profundidade. Por outro lado, em sistemas mais conservacionistas existe a tendência de concentração dos nutrientes próximos à superfície, havendo assim a necessidade de subdivisão da camada costumeiramente adotada em preparo convencional, que é de 0 a $20 \mathrm{~cm}$.

Para amostragem do solo foram traçados planos de coleta que consistiram na divisão das áreas de estudo em quatro subáreas de acordo com a atividade desenvolvida (cultivos orgânico, em conversão para orgânico, convencional e mata nativa). Cada subárea estudada dentro das áreas foi dividida em três quadrantes, considerados como repetições, sendo abertas quatro trincheiras de $30 \times 30 \times 30 \mathrm{~cm}$ em cada um desses três quadrantes e coletadas quatro subamostras para cada camada, que após homogeneizadas constitui-se em uma amostra composta para cada um dos três quadrantes e para cada camada e, consequentemente, três amostras compostas para cada camada e seis amostras compostas para cada subárea estudada.

As características físicas estudadas foram: Ds, Dp e PT. Dentre as características químicas do solo foram estudadas: $\mathrm{pH}$, teores $\mathrm{P}, \mathrm{K}, \mathrm{Ca}, \mathrm{Mg}, \mathrm{Na}, \mathrm{H}+\mathrm{Al}$ e $\mathrm{CO}$. Quanto às características biológicas foram estudadas MOPL, CMOLP e RE.

A Ds foi obtida pela razão entre o peso da amostra seca a $105{ }^{\circ} \mathrm{C}$ e o volume do cilindro, a Dp pelo método do balão volumétrico com água fervente e a PT pela expressão: $\mathrm{PT}=1-\left(\frac{\mathrm{Ds}}{\mathrm{Dp}}\right) \times 100$, onde $\mathrm{Ds}=$ densidade do solo e $\mathrm{Dp}=$ densidade de partículas (EMBRAPA, 1997).

As propriedades químicas foram: $\mathrm{pH}$ em água $(1: 2,5)$, carbono orgânico $(\mathrm{CO})$, bases trocáveis $\left(\mathrm{Ca}^{2+}, \mathrm{Mg}^{2+}, \mathrm{Na}^{+}\right.$e $\left.\mathrm{K}^{+}\right), \mathrm{P}$ disponível e acidez potencial $(\mathrm{H}+\mathrm{Al})$ determinadas conforme Embrapa
(1997), assim como estimadas a soma de bases (SB), a capacidade de troca de cátions (CTC) e a saturação por bases (V\%).

Quanto as propriedades biológicas, MOPL foi obtida por flotação em água (BURAK et al., 2011). As frações leves separadas das amostras de solo foram analisadas quanto aos teores de $\mathrm{C}$, de acordo com metodologias descritas por Embrapa (1997). A $\mathrm{RE}$ foi obtida semelhantemente àquela descrita em Araujo et al. (2008).

Os efeitos dos sistemas de uso do solo sobre suas características físicas, químicas e biológicas, em cada camada, foram avaliados a partir da análise de variância, seguindo-se um delineamento inteiramente casualizado, com três repetições. As médias foram comparadas pelo teste Tukey, até o nível de 5\% de probabilidade. Os dados também foram submetidos à Análise de Componentes Principais (ACP).

A Análise de Componentes Principais objetivou sintetizar a variação multidimensional dos dados, ordenando-os nos eixos, de acordo com suas similaridades em termos das variáveis utilizadas (TER BRAAK, 1986).

As amostras (sistemas de cultivo) e as variáveis (características físicas, químicas e biológicas) foram transformadas em coordenadas (escores), que correspondem a sua projeção nos eixos de ordenação, ou autovalores ("eigenvectors"), representando o peso de cada variável sobre o eixo, as quais podem ser vistas como equivalentes ao grau de correlação destes com o eixo em questão.

Apenas os dois primeiros componentes principais foram utilizados, por serem considerados suficientes para explicar os dados e facilitarem a interpretação do gráfico em duas dimensões (GOMES et al., 2004). Os componentes principais foram escolhidos de forma a reterem mais de $60 \%$ da variabilidade total dos dados, sendo que cada componente principal teve suas variáveis mais representativas, sendo estas os mais altos autovalores em módulo até o limite inferior de 1/4 do mais elevado autovalor em módulo.

\section{RESULTADOS E DISCUSSÃO}

Os solos de mata utilizados como referência nas áreas estudadas, com exceção do solo da área II, apresentaram menores valores de densidades do solo (Ds), mostrando que o uso antrópico aumentou a compactação do solo, principalmente nos solos sob cultivo convencional que apresentaram, de modo geral, os maiores valores de Ds (Tabela 1). 
Tabela 1. Médias $(n=3)$ dos atributos físicos de amostras de solo coletadas nos municípios de Areia (Área I), Remígio (Área II) e Lagoa Seca (Área III), mesorregião do Agreste da Paraíba, em diferentes camadas e sistemas de cultivo.

\begin{tabular}{|c|c|c|c|c|c|c|c|c|c|c|c|c|}
\hline \multirow{3}{*}{$\begin{array}{l}\text { Cam. } \\
(\mathrm{cm})\end{array}$} & \multicolumn{12}{|c|}{ Sistema de Cultivo } \\
\hline & \multicolumn{4}{|c|}{ Área I (PVAe) } & \multicolumn{4}{|c|}{ Área II (RRe) } & \multicolumn{4}{|c|}{ Área III (RRe) } \\
\hline & Mata & $\mathrm{CO}$ & CEC & $\mathrm{CC}$ & Mata & $\mathrm{CO}$ & CEC & $\mathrm{CC}$ & Mata & $\mathrm{CO}$ & CEC & $\mathrm{CC}$ \\
\hline & & & & & $-\mathrm{De}$ & ade do & $0, \mathrm{~g} \mathrm{cn}$ & & & & & \\
\hline $0-10$ & $1,1 \mathrm{c}$ & $1,2 \mathrm{~b}$ & $1,2 \mathrm{~b}$ & $1,3 \mathrm{a}$ & $1,0 \mathrm{~b}$ & $1,1 \mathrm{~b}$ & $1,4 \mathrm{a}$ & $1,4 \mathrm{a}$ & $1,0 \mathrm{c}$ & $1,1 \mathrm{~b}$ & $1,2 \mathrm{~b}$ & $1,4 \mathrm{a}$ \\
\hline $10-20$ & $1,1 \mathrm{c}$ & $1,2 \mathrm{~b}$ & $1,2 \mathrm{~b}$ & $1,3 \mathrm{a}$ & $1,1 \mathrm{~b}$ & $1,2 \mathrm{~b}$ & $1,4 \mathrm{a}$ & $1,4 \mathrm{a}$ & $1,0 \mathrm{c}$ & $1,2 \mathrm{~b}$ & $1,3 \mathrm{~b}$ & $1,4 \mathrm{a}$ \\
\hline $0-10$ & $2.7 \mathrm{a}$ & $2.6 \mathrm{a}$ & $2.6 \mathrm{a}$ & $2.8 \mathrm{a}$ & \multicolumn{4}{|c|}{-- Densidade de partículas, $\mathrm{g} \mathrm{cm}^{-3}$} & $2,6 \mathrm{a}$ & $2,7 \mathrm{a}$ & $2,8 \mathrm{a}$ & $2.7 \mathrm{a}$ \\
\hline \multirow[t]{2}{*}{$10-20$} & $2,7 \mathrm{a}$ & $2,6 \mathrm{a}$ & $2,7 \mathrm{a}$ & $2,7 \mathrm{a}$ & $2,7 \mathrm{a}$ & $2,7 \mathrm{a}$ & $2,7 \mathrm{a}$ & $2,7 \mathrm{a}$ & $2,6 \mathrm{a}$ & $2,7 \mathrm{a}$ & $2,7 \mathrm{a}$ & $2,7 \mathrm{a}$ \\
\hline & & & & & \multicolumn{4}{|c|}{ Porosidade total, $\mathrm{m} \mathrm{m}^{-3}$} & & & & \\
\hline $0-10$ & $0,6 \mathrm{a}$ & $0,5 \mathrm{~b}$ & $0,5 \mathrm{~b}$ & $0,4 \mathrm{c}$ & $0,6 \mathrm{a}$ & $0,5 \mathrm{~b}$ & $0,4 \mathrm{c}$ & $0,4 \mathrm{c}$ & $0,6 \mathrm{a}$ & $0,5 \mathrm{~b}$ & $0,5 \mathrm{~b}$ & $0,5 \mathrm{~b}$ \\
\hline $10-20$ & $0,6 \mathrm{a}$ & $0,5 \mathrm{~b}$ & $0,5 \mathrm{~b}$ & $0,5 \mathrm{~b}$ & $0,6 \mathrm{a}$ & $0,5 \mathrm{~b}$ & $0,5 \mathrm{~b}$ & $0,5 \mathrm{~b}$ & $0,6 \mathrm{a}$ & $0,5 \mathrm{~b}$ & $0,4 \mathrm{c}$ & $0,4 \mathrm{c}$ \\
\hline
\end{tabular}

Médias seguidas de mesma letra, nas linhas, para uma mesma área e camada não diferem entre si pelo teste de Tukey a 5\% de probabilidade; Cam.: Camada; CO: Cultivo orgânico; CEC: Cultivo em conversão para orgânico; CC: Cultivo convencional; (PVAe) e (RRe), respectivamente: Argissolo Vermelho Amarelo e Neossolo Regolítico.

Os resultados da Ds encontrados neste trabalho estão de acordo com os obtidos por Portugal et al. (2010), os quais constataram valores médios significativamente maior em áreas cultivadas, comparadas com o solo sob floresta. Avaliando atributos relacionados à qualidade do solo sob diferentes usos e manejos Chaves et al. (2012) constataram que as áreas amostrais que se encontravam sob exploração agrícola apresentaram maiores valores de Ds, em comparação com a área de cerrado nativo destituída de qualquer uso antrópico.

Independente da camada estudada, os solos submetidos ao cultivo orgânico apresentaram menores valores de Ds em relação aos solos sob cultivo convencional (Tabela 1). Esses maiores valores de Ds encontrados nas áreas sob cultivo convencional em relação às áreas sob cultivo orgânico pode ser explicado pela ação de máquinas e pelo uso intensivo dos solos das áreas convencionais, ocasionando maior pressão no solo e consequentemente maior valor de Ds, semelhantemente ao descrito por Mentges et al. (2010).

A constatação de redução da Ds nos sistemas de cultivo orgânico em relação aos sistemas de cultivo convencional neste trabalho indica melhoria na qualidade física do solo decorrente, possivelmente, do maior aporte de matéria orgânica nesses sistemas e da atividade da fauna e das raízes, as quais atuam na formação de bioporos. A baixa Ds encontrada em cultivos orgânicos deve-se, também, às partículas orgânicas cuja densidade for $<1,0 \mathrm{~g} \mathrm{~cm}^{-3}$ e ao tipo de arranjamento dessas partículas (COSTA; ABRÃO 1996). Ainda segundo os autores, a matéria orgânica associada a solos de textura arenosa, a exemplo dos predominantes nas áreas desse estudo, imprime maior velocidade de decomposição, contribuindo para aumentar a formação das substâncias húmicas, favorecendo a agregação das partículas e, consequentemente, a redução da Ds.

Apesar da constatação de alterações significativas dos valores Ds em função dos cultivos, nas diferentes áreas estudadas, os valores encontrados foram menores que o valor crítico do crescimento radicular para solos de textura arenosa, $1,7 \mathrm{~g} \mathrm{~cm}^{-3}$ (PAPA et al., 2011), não sendo esses valores considerados como limitantes ou com potencial para prejudicar o crescimento radicular e, consequentemente, o pleno desenvolvimento das culturas.

Diferentemente da Ds, para todas as áreas estudadas a Dp não foi influenciada pelos sistemas de cultivos utilizados (Tabela 1). De acordo com Rühlmann et al. (2006), a variável Dp praticamente não é influenciada por alterações no manejo por estar mais relacionada à constituição mineralógica e com o conteúdo de matéria orgânica do solo.

A baixa Ds encontrada no solo de mata se refletiu nos valores de porosidade total desse sistema, em todas as áreas e camadas estudadas (Tabela 1), confirmando assim a relação inversa existente entre densidade do solo e porosidade total (CHAVES et al., 2012). De acordo com Bertol et al. (2004), solos menos submetidos a processos de perturbações antrópicas, a exemplo dos solos sob matas e sob cultivos orgânicos, têm porosidade total superior aqueles apresentados nos sistemas convencionais de cultivo. Verifica-se, também, diferença significativa para a porosidade total do solo entre os sistemas de produção orgânico e convencional, nas camadas de 0-10 $\mathrm{cm}$ das áreas I e II e na camada de 10-20 cm da área III (Tabela 1). Esses resultados discordam daqueles obtidos por Lima et al. (2007), no qual não encontraram diferenças significativas para a porosidade total do solo em função dos tipos de cultivos adotados.

Com exceção da área I na camada de $0-10 \mathrm{~cm}$ e da área II na camada de $10-20 \mathrm{~cm}$ em todas as demais áreas e independente da camada estudada os maiores valores de $\mathrm{pH}$ do solo foram observados para os solos manejados nos sistemas de cultivo em conversão para orgânico e convencional, tendo os solos sob mata, de modo geral, apresentado os menores valores (Tabela 2). Esses dados mostram-se condizentes, pois os solos sob mata geralmente apresentam menores valores de $\mathrm{pH}$, principalmente para estes tipos de solo estudados na presente pesquisa, os 
quais devido a sua mineralogia são dependentes de pH (dissociação de $\mathrm{OH})$. Além disso, a mineralização da matéria orgânica e os exsudatos ácidos libera- dos pelas raízes das plantas também contribuem para aumentar a acidez do solo.

Tabela 2. Médias $(n=3)$ dos atributos químicos de amostras de solo coletadas nos municípios de Areia (Área I), Remígio (Área II) e Lagoa Seca (Área III), mesorregião do Agreste Paraibano, em diferentes camadas e sistemas de cultivo.

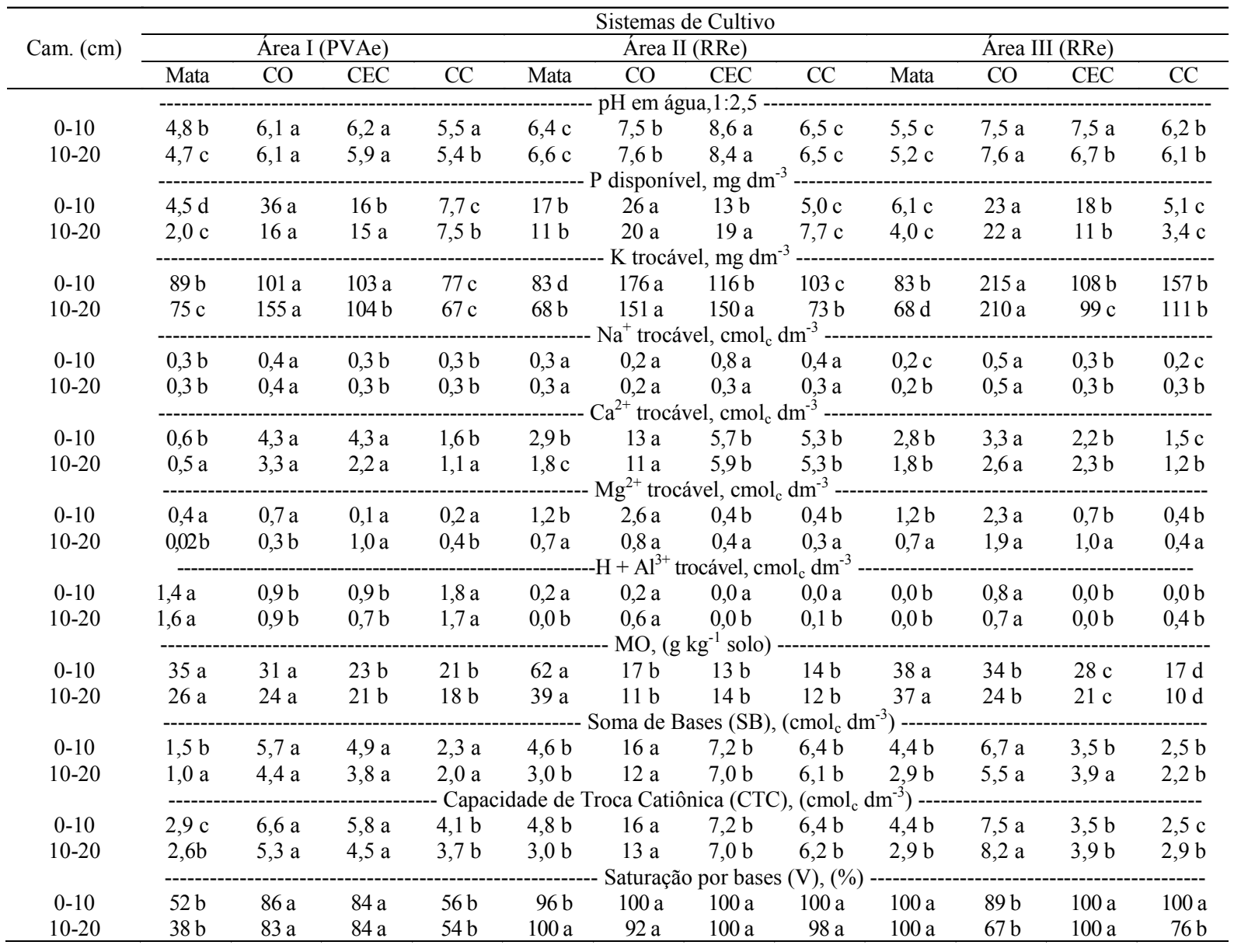

Médias seguidas de mesma letra, nas linhas, para uma mesma área e camada não diferem entre si pelo teste de Tukey a 5\% de probabilidade; Cam.: Camada; CO: Cultivo orgânico; CEC: Cultivo em conversão para orgânico; CC: Cultivo convencional; (PVAe) e (RRe), respectivamente: Argissolo Vermelho Amarelo e Neossolo Regolítico.

Os valores mais elevados de $\mathrm{pH}$ dos solos sob cultivo em conversão para orgânico e orgânico comparado aos solos sob cultivo convencional podem estar relacionados a adição de cátions básicos ao solo em função do aporte contínuo de material orgânico ao solo nesses sistemas de cultivo, sugerindo que a adição do composto orgânico contribuiu para anular as cargas positivas da matriz mineral do solo pela adsorção específica de ânions orgânicos, resultando em baixa acidificação do solo. Por outro lado, a constante utilização de adubos nitrogenados solúveis de fontes amoniacais pelos produtores das áreas sob cultivo convencional deste estudo contribuíram para a obtenção de valores mais baixos de $\mathrm{pH}$ em relação aos sistemas em conversão para orgânico e orgânico.

Com exceção do solo de mata da área II na camada de 0-10 cm, o qual não diferiu estatisticamente do solo sob cultivo em conversão para orgânico, em todas as áreas, independente da camada, os teores de $\mathrm{P}$ disponíveis foram estatisticamente maiores nos solos sob cultivo orgânico e em conversão para orgânico (Tabela 2), o que pode estar relacionado com a forma de manejo adotada nesses sistemas, com aplicação contínua de esterco e composto orgânico, principalmente devido o tipo de produção utilizada nas áreas ser o de hortaliças, as quais demandam grandes quantidades de nutrientes em pequeno espaço de tempo.

O uso complementar do adubo da independência (tipo de compostagem), biofertilizante, compostagem, húmus de minhoca, biomassa foliar e urina de vaca pelos produtores das áreas, de acordo com as possibilidades e conhecimentos de cada um, também contribui para os aumentos nos teores de $\mathrm{P}$ dos solos nestes sistemas de cultivo, visto que de acordo com Souza e Resende (2003) a utilização de grandes quantidades de esterco no cultivo intensivo de hortaliças provoca incrementos de nutrientes no solo, 
principalmente de fósforo.

Incrementos nos teores de $\mathrm{P}$ no solo em decorrência do emprego de sistemas de cultivo orgânicos em comparação a sistemas de cultivo convencional também foram registrados por Clark et al. (1998).

Contudo, os maiores teores de $\mathrm{P}$ disponível nos solos sob cultivo em conversão para orgânico e orgânico e com base nos teores de argila da fração granulométrica das áreas avaliadas, de $13 \%$ na área I, 6\% na área II e 4\% na área III, os teores deste nutriente nos solos sob mata nas áreas, em ambas as camadas, foram classificados, de acordo com Ribeiro et al. (1999), como muito baixo (área I), baixo (área II) e muito baixo (área III) (Tabela 2). Nos solos sob cultivo orgânico esses teores foram classificados como bom, médio e médio (para as áreas I, II e III, respectivamente, na camada de $0-10 \mathrm{~cm})$ e baixo, médio e médio (para as áreas I, II e III, respectivamente, na camada de $10-20 \mathrm{~cm}$ ).

Independente da camada, os teores de $\mathrm{P}$ foram classificados como baixo nas áreas sob cultivo em conversão para orgânico e muito baixo nas áreas sob cultivo convencional. Isto evidencia a baixa disponibilidade natural de $\mathrm{P}$ nos solos brasileiros, não sendo diferente nos solos das áreas estudadas, que mesmo apresentando valores significativamente maiores nos solos sob cultivo orgânico e em conversão para orgânico em relação ao sistema convencional e a mata indicou a necessidade de um manejo adequado da adição de $\mathrm{P}$ para garantir teores ideias do nutriente no solo e, consequentemente, obtenção de produtividades satisfatórias nestes sistemas de produção.

Por outro lado, os teores de $\mathrm{P}$ encontrados nas áreas sob cultivo (Tabela 2) indicam que a adição sistemática de materiais orgânicos no sistema de cultivo orgânico, e em menor magnitude no sistema convencional, tem se mostrado capaz de proporcionar acréscimos substanciais nos teores desse nutriente em relação aos teores originalmente encontrados sob condições não antropizadas, a exemplo da testemunha (mata) a qual apresenta valores médios entre as áreas estudadas de 9,2 para a camada de 0-10 e 5,7 para a camada $10-20 \mathrm{~cm}$.

De modo geral, os menores valores de nutrientes nos solos foram encontrados sob mata e sob cultivo convencional (Tabela 2), o que em parte se explicam pelo fato de que nos solos sob mata a maioria dos nutrientes está alocada na vegetação (SILVA et al., 2013) e, no cultivo convencional, devido a, possivelmente, ausência de práticas de manejo que favoreçam a manutenção e/ou melhoria da fertilidade do solo, como a adição de matéria orgânica e a reposição dos nutrientes exportados pelas produções das culturas cultivadas e/ou perdidos pela erosão e lixiviação.

Considerando-se os sistemas de manejo do solo, observou-se que os solos sob o sistema de cultivo orgânico, em relação aos solos submetidos ao sistema convencional de manejo, apresentaram médias estatisticamente maiores para os teores de $\mathrm{K}$, Ca (com exceção da área I na camada de 10-20 cm), Mg (nas áreas II e III, na camada de 0-10 cm), SB (com exceção da área I), CTC e V\% (área I) (Tabela 2), sendo que, sob cultivo orgânico e independente das áreas e das camadas estudadas, o solo apresentou fertilidade média adequada, com valores de K classificados como muito bom, Ca como muito bom nas áreas I e II e bom na área III, $\mathrm{Mg}$ variando de baixo (área I, na camada de $10-20 \mathrm{~cm}$ ) a muito bom (áreas II e III, na camada de $0-10 \mathrm{~cm}), \mathrm{SB}$ variando de bom (áreas I e III) a muito bom (área II), CTC variando de médio (áreas I e III) a muito bom (área II) e V\% para todas as áreas como muito bom (RIBEIRO et al., 1999).

Os resultados de $\mathrm{Ca}$ e $\mathrm{K}$ observados neste trabalho para os solos sob sistemas conservacionistas em relação ao solo sob cultivo convencional são corroborados pelos resultados obtidos por Bilibio et al. (2010). Os valores de SB e de CTC observados nos solos sob sistemas de cultivos orgânicos e em conversão para orgânico indicam que a matéria orgânica aplicada ou o tempo de manejo orgânico foi suficiente para alterar a capacidade dos solos em reter cátions.

O menor teor de cátions trocáveis nos solos sob cultivo convencional em relação aos solos sob cultivo orgânico pode ser atribuído às perdas de nutrientes pouco retidos nos sítios de troca, em função principalmente dos menores teores de matéria orgânica, devendo, dessa forma, ser manejados com critérios rigorosos, a fim de atingirem seu máximo potencial produtivo, sem que provoque a sua degradação. Por outro lado, os maiores valores obtidos para os teores de nutrientes nos solos sob cultivo orgânico refletem o manejo e o histórico de adição de nutrientes, em função do aporte continuo de esterco e materiais orgânicos de diversas origens nesses sistemas de cultivo, indicando, também, que o aporte desses materiais e o tempo de manejo orgânico desses solos foram suficientes para alterar a capacidade dos solos em reter cátions, tendo em vista a similaridade da fração granulométrica dos solos das áreas estudadas, o que se refletiu em maior fertilidade desses solos em comparação aos solos sob cultivo convencional.

Niero et al. (2010), avaliando a sustentabilidade de práticas de manejo do solo, constataram menores valores de fertilidade do solo para o cultivo convencional em relação aos demais tipos de cultivo estudados. Lourente et al. (2011), estudando o efeito do uso e manejo sobre atributos químicos, físicos e microbiológicos do solo, tendo também como referência a mata nativa, constataram que dentre os sistemas de manejo do solo estudados o sistema convencional apresentou valores médios de fertilidade do solo estatisticamente menores.

Com exceção da área I, em que o solo sob mata não diferiu estatisticamente do solo sob cultivo orgânico em relação ao teor de matéria orgânica, 
para as demais áreas os teores de matéria orgânica do solo foram estatisticamente maiores nos solos sob mata, nas duas camadas avaliadas, indicando que a retirada da mata e a utilização agrícola dos solos reduziram os teores de $\mathrm{C}$ orgânico desses solos. Esse resultado está de acordo com Portugal et al. (2010), segundo os quais há um declínio no estoque de matéria orgânica após a conversão de florestas nativas em sistemas agrícolas, sendo essa redução atribuída ao aumento da erosão do solo, aos processos mais acelerados de mineralização da matéria orgânica do solo e as menores quantidades de aportes orgânicos em sistemas manejados comparativamente às florestas nativas.

Ao comparar os sistemas de manejo do solo, constatou-se que à exceção da área II os solos sob sistema de cultivo orgânico, em relação aos solos submetidos ao sistema de cultivo convencional, nas duas camadas, apresentaram médias estatisticamente maiores para os teores de MO (Tabela 2). Esses resultados demonstram que os sistemas de cultivo convencionais, caracterizados pelo intenso revolvimento do solo e pelo uso de elevadas quantidades de fertilizantes minerais e pesticidas, contribuem mais intensamente para as reduções de MO do solo comparado aos sistemas de cultivo orgânico. Kamiyama et al. (2011), testando as hipóteses de que os produtores orgânicos têm maior percepção ambiental e adotam práticas conservacionistas, e a de que a agricultura orgânica resulta em melhor qualidade do solo, constataram que os sistemas de produção orgânicos avaliados apresentaram maior teor de matéria orgânica comparativamente aos sistemas convencionais.

Pelos resultados obtidos, observa-se também que os maiores teores de matéria orgânica observados nos solos sob sistema de cultivo orgânico em relação aos solos sob sistemas de cultivo convencio- nal foram acompanhados de maiores valores de CTC para estes solos (Tabela 2), o que já era esperado, visto que do ponto de vista químico o teor de matéria orgânica está estreitamente relacionado a CTC do solo (BILIBIO et al., 2010).

Os maiores valores de matéria orgânica particulada leve (MOPL) foram observados para os solos de mata, em todas as áreas e camadas estudadas (Tabela 3). Esses dados mostram-se coerentes, visto que a substituição de floresta natural por agricultura resulta em uma diminuição no conteúdo de MO particulada, refletindo-se também sobre a MOPL (LIMA et al., 2008).

Ao comparar os solos sob sistemas de cultivo orgânico e convencional foram observados os maiores valores de MOPL para os solos sob cultivo orgânico (Tabela 3). Esse fato pode ser atribuído a influência do manejo mais adequado dos solos sob sistema de cultivo orgânico, com aporte contínuo de matéria orgânica, dando condições para o aumento no estoque de MOPL nesses solos em relação aos solos submetidos ao cultivo convencional, onde o aporte de material orgânico ao solo é mínimo.

Variações do conteúdo dos teores de MOPL podem ser resultantes das mudanças na quantidade $\mathrm{e}$ qualidade dos resíduos vegetais que foram adicionados ao solo, da relação entre a entrada por superfície e subsuperfície destes resíduos e, principalmente, das diferentes formas de manejo adotadas (BARRETO et al., 2008). Logo, o acúmulo de MOPL pode representar maior fornecimento de substrato a ser utilizado como fonte de energia para o crescimento microbiano, levando a liberação de nutrientes por meio da ciclagem da biomassa microbiana e favorecendo a recuperação do equilíbrio biológico do solo e de sua qualidade.

Tabela 3. Valores médios $(n=3)$ de matéria orgânica particulada leve (MOPL), carbono da matéria orgânica particulada leve (CMOPL) e respiração edáfica (RE) de amostras de solos coletadas nos municípios de Areia (Área I), Remígio (Área II) e Lagoa Seca (Área III), mesorregião do Agreste Paraibano, em diferentes camadas e sistemas de cultivo.

\begin{tabular}{|c|c|c|c|c|c|c|c|c|c|c|c|c|}
\hline \multirow{2}{*}{$\begin{array}{l}\text { Cam. } \\
(\mathrm{cm})\end{array}$} & \multicolumn{12}{|c|}{ Sistema de Cultivo } \\
\hline & \multicolumn{4}{|c|}{ Área I (AVA) } & \multicolumn{4}{|c|}{ Área II (NR) } & \multicolumn{4}{|c|}{ Área III (NR) } \\
\hline & Mata & $\mathrm{CO}$ & CEC & $\mathrm{CC}$ & Mata & $\mathrm{CO}$ & CEC & $\mathrm{CC}$ & Mata & $\mathrm{CO}$ & CEC & $\mathrm{CC}$ \\
\hline $0-10$ & $34 \mathrm{a}$ & $27 \mathrm{~b}$ & $15 \mathrm{c}$ & $11 \mathrm{c}$ & $35 \mathrm{a}$ & $27 \mathrm{~b}$ & $\begin{array}{c}\mathrm{Kg} \text { so } \\
18 \mathrm{c}\end{array}$ & $9,9 \mathrm{~d}$ & $35 \mathrm{a}$ & $28 \mathrm{~b}$ & $18 \mathrm{c}$ & $10 \mathrm{~d}$ \\
\hline $10-20$ & $30 \mathrm{a}$ & $22 \mathrm{~b}$ & $14 \mathrm{c}$ & $11 \mathrm{c}$ & $31 \mathrm{a}$ & $22 \mathrm{~b}$ & $14 \mathrm{c}$ & $8,9 \mathrm{~d}$ & $35 \mathrm{a}$ & $24 \mathrm{~b}$ & $12 \mathrm{c}$ & $9,4 \mathrm{c}$ \\
\hline $0-10$ & $14 \mathrm{a}$ & $11 \mathrm{~b}$ & $4,5 \mathrm{c}$ & $3,0 \mathrm{c}$ & $13 \mathrm{a}$ & $11 \mathrm{~b}$ & $\begin{array}{l}\mathrm{g} \mathrm{kg} \\
4,7 \mathrm{c}\end{array}$ & $3,1 \mathrm{~d}$ & $12 \mathrm{a}$ & $10 \mathrm{~b}$ & $4,6 \mathrm{c}$ & $3,1 \mathrm{~d}$ \\
\hline $10-20$ & $14 \mathrm{a}$ & $10 \mathrm{~b}$ & $4,0 \mathrm{c}$ & $3,5 \mathrm{c}$ & $14 \mathrm{a}$ & $9,2 \mathrm{~b}$ & $4,0 \mathrm{c}$ & $3,8 \mathrm{c}$ & $13 \mathrm{a}$ & $9,8 \mathrm{~b}$ & $4,3 \mathrm{c}$ & $3,4 \mathrm{~d}$ \\
\hline $\begin{array}{c}0-10 \\
10-20\end{array}$ & $\begin{array}{l}51 \mathrm{~d} \\
50 \mathrm{~d}\end{array}$ & $\begin{array}{l}95 \mathrm{a} \\
84 \mathrm{a}\end{array}$ & $\begin{array}{l}71 \mathrm{~b} \\
68 \mathrm{~b}\end{array}$ & $\begin{array}{l}59 \mathrm{c} \\
58 \mathrm{c}\end{array}$ & $\begin{array}{l}50 \mathrm{~d} \\
50 \mathrm{~d}\end{array}$ & $\begin{array}{l}95 \mathrm{a} \\
80 \mathrm{a}\end{array}$ & $\begin{array}{l}71 \mathrm{~b} \\
71 \mathrm{~b}\end{array}$ & $\begin{array}{l}59 \mathrm{c} \\
57 \mathrm{c}\end{array}$ & $\begin{array}{l}51 \mathrm{~d} \\
51 \mathrm{~d}\end{array}$ & $\begin{array}{l}95 \mathrm{a} \\
81 \mathrm{a}\end{array}$ & $\begin{array}{l}73 \mathrm{~b} \\
69 \mathrm{~b}\end{array}$ & $\begin{array}{l}58 \mathrm{c} \\
57 \mathrm{c}\end{array}$ \\
\hline
\end{tabular}

Médias seguidas de mesma letra, nas linhas, para uma mesma área e camada não diferem entre si pelo teste de Tukey a 5\% de probabilidade; Cam.: Camada; CO: Cultivo orgânico; CEC: Cultivo em conversão para orgânico; CC: Cultivo convencional; (AVA) e (NR), respectivamente: Argissolo Vermelho Amarelo e Neossolo Regolítico.

De modo semelhante ao teor de MOPL, constatou-se maior teor de C presente na MOPL nos solos sob mata (Tabela 3). Verificou-se também que o teor de $\mathrm{C}$ presente na MOPL nos solos sob sistema de cultivo orgânico foi aproximadamente três vezes maior que o teor obtido nos solos sob sistemas de cultivo convencional. Para Leite et al. (2003), sistemas que oferecem maior cobertura do solo (a exem- 
plo da mata nativa) e que proporcionam maior aporte de carbono no solo (a exemplo do cultivo orgânico), em consequência de uma ciclagem de nutrientes mais intensa, geralmente apresentam teores mais elevados de MOPL e de CMOPL. Diante desses resultados, constata-se que os sistemas de cultivo orgânico e de mata proporcionaram maiores quantidades de MOPL e CMOPL, indicando menores perdas de carbono nesses sistemas, podendo traduzir-se em uma recuperação da qualidade do solo. Dessa forma, a MOPL e o CMOPL podem vir a ser utilizadas como indicadores de alterações resultantes do manejo do solo nas áreas sob estes sistemas de cultivo.

A respiração edáfica foi influenciada pelos sistemas de cultivo avaliados (Tabela 3). Independente das camadas analisadas é observada uma maior atividade da biomassa microbiana nos sistemas de cultivo orgânicos em relação aos demais. Resultados semelhantes aos verificados neste estudo foram encontrados por Sampaio et al. (2008), os quais avaliaram a microbiota do solo em sistemas de cultivo convencional e orgânico e observaram maior atividade microbiana no solo sob cultivo orgânico.

Da mesma forma, Martins Neto et al. (2010) avaliando por meio de indicadores bióticos e abióticos a qualidade do solo, assim como a nutrição de cafeeiros cultivados sob manejos convencional e orgânico, constataram que a respiração edáfica nos sistemas de manejo orgânico foi superior quando comparada aos sistemas manejados convencionalmente, nos dois períodos do ano avaliados.

Verifica-se, também, que os solos da área de mata apresentaram os menores valores de respiração edáfica em relação aos demais, sugerindo uma maior estabilidade da mata nativa, pois baixa respiração basal é indicativo de ecossistemas mais estáveis (SAMPAIO et al., 2008). Por outro lado, a maior proximidade dos valores de respiração edáfica dos solos sob cultivo convencional em relação aos solos da área de mata verificada neste trabalho não se reflete em maior estabilidade desses solos em relação aos demais sistemas de cultivo avaliados, mas sim às diferenças nos aportes de carbono (C), incluindo quantidade e qualidade, já que nos sistemas orgânicos os microrganismos heterotróficos são responsáveis por maiores taxas respiratórias (MARTINS NETO et al., 2010).

$\mathrm{O}$ resultado da análise de componentes principais (ACP) conjunta para os atributos físicos, químicos e biológicos do solo indicou dois componentes principais respondendo por $64,6 \%$ da variabilidade total dos dados, onde o Componente Principal 1 explicou $40 \%$ e o Componente Principal 2 explicou $24,6 \%$ da variabilidade total dos dados (Tabela 4).

Tabela 4. Autovalores da análise de componentes principais (ACP) para os atributos físicos, químicos e biológicos de solos da Mesorregião do Agreste Paraibano.

\begin{tabular}{ccccc}
\hline $\begin{array}{c}\text { Componente } \\
\text { Principal }\end{array}$ & Autovalores & Diferença & \% total da variação dos dados & Acumulativo \\
\hline 1 & 8,80 & & 40,0 & 40,0 \\
2 & 5,42 & 3,38 & 24,6 & 64,6 \\
\hline
\end{tabular}

Analisando os valores modulares dos loadings da Tabela 5 e aplicando o critério da escolha em que cada componente principal tem suas variáveis mais representativas que são os mais elevados autovalores em módulo até o limite inferior de 1/4 do mais alto autovalor em módulo, verifica-se que para o Componente Principal 1 as variáveis que explicaram mais fortemente a variação dos dados foram Ds, PT, MO, CTC, MOPL e CMOPL. Para o Componente Principal 2, o pH, os teores médios de $\mathrm{Ca}$ e $\mathrm{H}+\mathrm{Al}$, a $\mathrm{SB}$ e a $\mathrm{RE}$ foram as variáveis que explicam mais fortemente a ordenação dos agrupamentos nesse eixo.

Tratando-se inicialmente do primeiro Componente Principal (CP 1), ao observar os valores reais da Tabela 5, verificou-se que as variáveis PT, MO, MOPL e CMOPL foram positivamente relacionadas com este eixo (CP 1), ao passo que as variáveis Ds e CTC foram negativamente relacionadas com o eixo em questão.

Quanto ao Componente Principal 2 (CP 2), verificou-se que as variáveis $\mathrm{pH}$, teores médios de $\mathrm{Ca}, \mathrm{SB}$ e $\mathrm{RE}$ foram positivamente relacionadas com este eixo, enquanto que a variável $\mathrm{H}+\mathrm{Al}$ foi negativamente relacionada com o eixo em questão.

Com base no exposto, analisando-se a Figura 1, observa-se que para o Componente Principal (CP 1), PT, MO, CTC, MOPL e CMOPL aumentaram da esquerda para a direita, enquanto que Ds e Dp diminuíram neste mesmo sentido. Para o segundo Componente Principal (CP 2), as variáveis $\mathrm{pH}$, teores médios de $\mathrm{Ca}, \mathrm{SB}, \mathrm{V}$ e RE aumentaram de baixo para cima, enquanto que a variável $\mathrm{H}+\mathrm{Al}$ diminuiu neste mesmo sentido. 
Tabela 5. Coeficientes de Estrutura da Análise de Componentes Principais (ACP) para os atributos físicos, químicos e biológicos de solos da Mesorregião do Agreste Paraibano.

\begin{tabular}{lcc}
\hline Variáveis & $\mathrm{CP} 1$ & $\mathrm{CP} 2$ \\
\hline Densidade do solo $(\mathrm{Ds}),\left(\mathrm{g} \mathrm{cm}^{-3}\right)$ & $\mathbf{- 0 , 3 0}$ & 0,09 \\
Densidade de partículas $(\mathrm{Dp}),\left(\mathrm{g} \mathrm{cm}^{-3}\right)$ & $-0,03$ & 0,07 \\
Porosidade total $(\mathrm{PT}),\left(\mathrm{m}^{3} \mathrm{~m}^{-3}\right)$ & $\mathbf{0 , 2 9}$ & $-0,05$ \\
$\mathrm{pH}$ & $-0,15$ & $\mathbf{0 , 3 1}$ \\
$\mathrm{P},\left(\mathrm{mg} \mathrm{dm}^{-3}\right)$ & 0,06 & 0,21 \\
$\mathrm{~K},\left(\mathrm{mg} \mathrm{dm}^{-3}\right)$ & 0,12 & 0,08 \\
$\mathrm{Ca},\left(\mathrm{cmol}_{\mathrm{c}} \mathrm{dm}^{-3}\right)$ & 0,17 & $\mathbf{0 , 2 9}$ \\
$\mathrm{Mg},\left(\mathrm{cmol}_{\mathrm{c}} \mathrm{dm}^{-3}\right)$ & 0,20 & 0,15 \\
$\mathrm{Na},\left(\mathrm{cmol}_{\mathrm{c}} \mathrm{dm}^{-3}\right)$ & 0,13 & $-0,03$ \\
$\mathrm{H}+\mathrm{Al},\left(\mathrm{cmol}_{\mathrm{c}} \mathrm{dm}^{-3}\right)$ & 0,08 & $\mathbf{- 0 , 3 6}$ \\
$\mathrm{MO},\left(\mathrm{g} \mathrm{kg}^{-1}\right)$ & $\mathbf{0 , 2 9}$ & 0,07 \\
$\mathrm{SB},\left(\mathrm{cmol}_{\mathrm{c}} \mathrm{dm}^{-3}\right)$ & 0,20 & $\mathbf{0 , 3 0}$ \\
$\mathrm{CTC},\left(\mathrm{cmol}_{\mathrm{c}} \mathrm{dm}^{-3}\right)$ & $\mathbf{- 0 , 2 3}$ & 0,25 \\
$\mathrm{~V},(\%)$ & $-0,06$ & 0,02 \\
MOPL, $\left(\mathrm{g} \mathrm{kg}^{-1}\right)$ & $\mathbf{0 , 2 7}$ & $-0,03$ \\
$\mathrm{CMOPL},\left(\mathrm{g} \mathrm{kg}^{-1}\right)$ & $\mathbf{0 , 2 7}$ & $-0,04$ \\
Respiração edáfica, $\left(\mathrm{mg} \mathrm{kg}^{-1}\right.$ solo) & $-0,14$ & $\mathbf{0 , 2 9}$ \\
\hline
\end{tabular}

MO: Matéria orgânica; SB: Soma de bases; CTC: Capacidade de troca de cátions; e V: Saturação por bases.

Esses resultados mostram que o sistema de cultivo orgânico apresentou grande probabilidade de autossustentabilidade do ponto de vista da qualidade física, química e biológica. Para tanto, é necessário que sejam tomadas medidas simples para a conserva- ção dos atributos físicos, químicos e biológicos mencionados neste estudo, já que alguns destes atributos apresentaram boa performance, como indicadores da qualidade do solo.

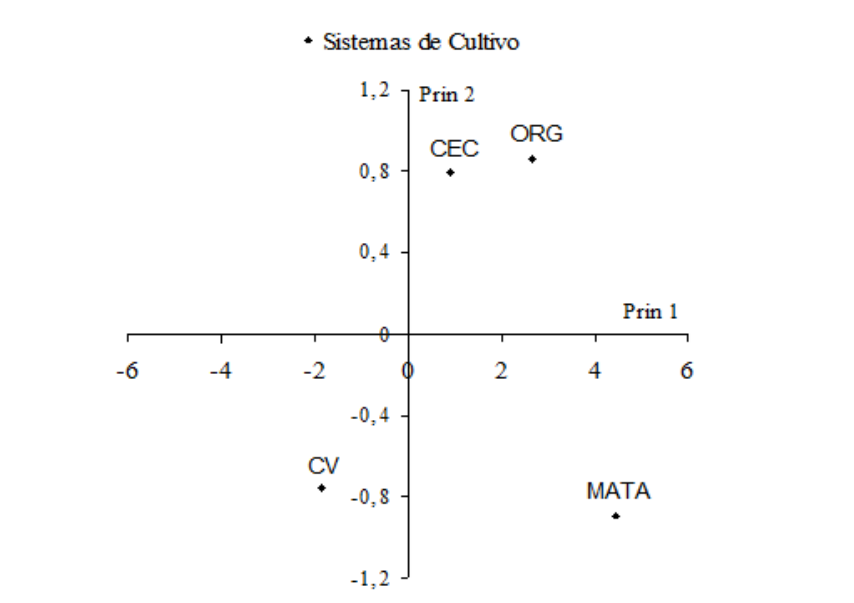

Figura 1. Escores dos sistemas de cultivo nos dois primeiros componentes principais obtidos com os atributos físicos, químicos e biológicos do solo. Cultivo orgânico (ORG), Cultivo em conversão para orgânico (CEC), Cultivo convencional (CV) e MATA.

\section{CONCLUSÕES}

Houve redução da Ds e aumento da PT no sistema de cultivo orgânico comparado aos sistemas de cultivo em conversão e convencional.

Os teores de $\mathrm{P}$ e de $\mathrm{CO}$, a $\mathrm{SB}$, a CTC, a MOPL, o CMOPL, a RE e o pH dos solos sob cultivo orgânico são superiores aos obtidos para os solos sob cultivo convencional.

O cultivo em bases orgânicas proporciona a manutenção da qualidade do solo em condições semelhantes e/ou melhores que a condição natural (mata nativa).

Para as condições deste trabalho, os indicadores mais adequados para analisar o cultivo em bases orgânicas foram Ds, PT, CO, MOPL, CMOPL, pH, $\mathrm{SB}, \mathrm{CTC}$ e RE.

\section{AGRADECIMENTOS}

A CAPES, pela bolsa de estudos concedida ao primeiro autor, e aos proprietários Vicente Félix da Silva (área I - Areia/PB), João Miranda Filho (área II - Remígio/PB) e Robson Alves Gertrudes (área III - Lagoa Seca/PB) pelo apoio e concessão das áreas para execução da pesquisa.

\section{REFERÊNCIAS}


ARAUJO, K. D. et al. Avaliação da atividade microbiana baseada na produção de $\mathrm{C}-\mathrm{CO}_{2}$ em uma área de caatinga no cariri paraibano. Revista Caatinga, Mossoró, v. 21, n. 3, p. 221-230, 2008.

BARRETO, A. C. et al. Fracionamento químico e físico do carbono orgânico total em um solo de mata submetido a diferentes usos. Revista Brasileira de Ciência do Solo, Viçosa, v. 32, n. 4, p. 1471-1478, 2008.

BAVOSO, M. A. et al. Preparo do solo em áreas de produção de grãos, silagem e pastejo: efeito na resistência tênsil e friabilidade de agregados. Revista Brasileira de Ciência do Solo, Viçosa, v. 34, n. 1, p. 227-234, 2010 .

BERTOL, I. et al. Propriedades físicas do solo sob preparo convencional e semeadura direta em rotação e sucessão de culturas, comparadas às do campo nativo. Revista Brasileira de Ciência do Solo, Viçosa, v. 28, n. 1, p. 155-163, 2004.

BILIBIO, W. D. et al. Atributos físicos e químicos de um Latossolo, sob diferentes sistemas de cultivo. Ciência e Agrotecnologia, Lavras, v. 34, n. 4, p. 817-822, 2010.

BURAK, D. L.; FONTES, M. P. F.; BECQUER, T. Microagregados estáveis e reserva de nutrientes em Latossolo Vermelho sob pastagem em região de Cerrado. Pesquisa Agropecuária Tropical, Goiânia, v. 41, n. 2 , p. 229-241, 2011.

CARNEIRO, M. A. C. et al. Atributos físicos, químicos e biológicos de solo de cerrado sob diferentes sistemas de uso e manejo. Revista Brasileira de Ciência do Solo, Viçosa, v. 33, n. 1, p. 147-157, 2009.

CHAVES, A. A. A. et al. Indicadores de qualidade de Latossolo Vermelho sob diferentes usos. Pesquisa Agropecuária Tropical, Goiânia, v. 42, n. 4, p. 446-454, 2012.

CLARK, M. S. et al. Changes in soil chemical properties resulting from organic and low-input farming practices. Agronomy Journal, Madison, v. 90, n. 5, p. 662-667, 1998.

COSTA, L. M.; ABRHÃO, W. A. P. Compactação e adensamento de solos relacionados às propriedades químicas, físicas e sedimentológicas. In: ALVAREZ, V., V. H.; FONTES, L. E. F.; FONTES, M. P. F. (Eds). O solo nos grandes domínios morfoclimáticos do Brasil e o desenvolvimento sustentado. 1. ed. Viçosa: UFV, 1996. p. 429-443.

DORAN, J. W.; PARKIN, T. B. Defining and assessing soil quality. In: DORAN, J. W. et al. (Eds.).
Defining soil quality for a sustainable environment. Madison: SSSA, 1994. v. 1, cap. 1, p. 3-21.

EMBRAPA. Centro Nacional de Pesquisas de Solos. Manual de métodos de análises de solo. 2 ed. rev. atual. Rio de Janeiro: 1997. 212 p.

EMBRAPA. Centro Nacional de Pesquisas de Solos. Sistema brasileiro de classificação de solos. 2 ed. Rio de Janeiro, 2006. 212 p.

GOMES, J. B. V. et al. Análise de componentes principais de atributos físicos, químicos e mineralógicos de solos do bioma Cerrado. Revista Brasileira de Ciência do Solo, Viçosa, v. 28, n. 1, p. 137-154, 2004.

KAMIYAMA, A. et al. Percepção ambiental dos produtores e qualidade do solo em propriedades orgânicas e convencionais. Bragantia, Campinas, v. 70, n. 1, p. 176-184, 2011.

LEITE, L. F. C. et al. Estoques totais de carbono orgânico e seus compartimentos em Argissolo sob floresta e sob milho cultivado com adubação mineral e orgânica. Revista Brasileira de Ciência do Solo, Viçosa, v. 27, n. 5, p. 821-832, 2003.

LIMA, A. M. N. et al. Frações da matéria orgânica do solo após três décadas de cultivo de eucalipto no Vale do Rio Doce-MG. Revista Brasileira de Ciência do Solo, Viçosa, v. 32, n. 3, p. 1053-1063, 2008.

LIMA, H. V. et. al. Indicadores de qualidade do solo em sistemas de cultivo orgânico e convencional no semi-árido cearense. Revista Brasileira de Ciência do Solo, Viçosa, v. 31, n. 5, p. 1085-1098, 2007.

LOURENTE, E. R. P. et al. Atributos microbiológicos, químicos e físicos de solo sob diferentes sistemas de manejo e condições de cerrado. Pesquisa Agropecuária Tropical, Goiânia, v. 41, n. 1, p. 20 $28,2011$.

MARINARI, S. et al. Chemical and biological indicators of soil quality in organic and conventional farming systems in Central Italy. Ecological Indicators, Amsterdam, v. 6, n. 4, p. 701-711, 2006.

MARTINS NETO, F. L. et al. Qualidade do solo e nutrição de plantas em sistemas de produção de café (Coffea arábica L.). Coffee Science, Lavras, v. 5, n. 3, p. 206-213, 2010.

MENTGES, M. I. et al. Propriedades físico-hídricas do solo e demanda energética de haste escarificadora em Argissolo compactado. Pesquisa Agropecuária Brasileira, Brasília, v. 45, n. 3, p. 315-321, 2010.

NIERO, L. A. C. et al. Avaliações visuais como índi- 
ce de qualidade do solo e sua validação por análises físicas e químicas em um Latossolo Vermelho distroférrico com usos e manejos distintos. Revista Brasileira de Ciência do Solo, Viçosa, v. 34, n. 4, p. 1271 $-1282,2010$.

PAPA, R. A. et al. Qualidade de Latossolos Vermelhos e Vermelho-Amarelos sob vegetação nativa de Cerrado. Pesquisa Agropecuária Tropical, Goiânia, v. 41, n. 4, p. 564-571, 2011.

PORTUGAL, A. F. et al. Propriedades físicas e químicas do solo em áreas com sistemas produtivos e mata na região da Zona da Mata Mineira. Revista Brasileira de Ciência do Solo, Viçosa, v. 34, n. 2, p. 575-585, 2010.

RIBEIRO, A. C.; GUIMARÃES, P. T. G.; ALVAREZ V., V. H. (Eds.). Recomendações para o uso de corretivos e fertilizantes em Minas Gerais. Viçosa: CFSEMG, 1999. 359 p.

RÜHLMANN, J.; KÖRSCHENS, M.; GRAEFE, J. A new approach to calculate the particle density of soils considering properties of the soil organic matter and the mineral matrix. Geoderma, Amsterdam, v. 130, n. 3-4, p. 272-283, 2006.

SAMPAIO, D. B. et al. Avaliação de indicadores biológicos de qualidade do solo sob sistemas de cultivo convencional e orgânico de frutas. Ciência e Agrotecnologia, Lavras, v. 32 , n. 2, p. 353-359, 2008.

SILVA, A. S. et al. Propriedades físicas e químicas em diferentes usos do solo no brejo paraibano. Revista Brasileira de Ciência do Solo, Viçosa, v. 37, n. 4, p. 1064-1072, 2013.

SOUZA, J. L.; RESENDE, P. Manual de horticultura orgânica. Viçosa: Aprenda Fácil, 2003. 564 p.

TER BRAAK, C. J. F. Canonical correspondence analysis: A new eigenvector technique for multivariate direct gradient analysis. Ecology, New York, v. 67, n. 5 , p. 1167-1179, 1986. 